BULL. AUSTRAL. MATH. SOC.

VOL. $29(1984), 123-127$.

\title{
REAL HYPERSURFACES OF A \\ COMPLEX PROJECTIVE SPACE II
}

\author{
SADAHIRO MAEDA
}

We consider a certain real hypersurface $M$ of a complex projective space. The purpose of this paper is to characterize $M$ in terms of Ricci curvatures.

\section{Introduction}

Let $P_{n}(\mathbb{C})$ be an $n$-dimensional complex projective space with Fubini-study metric of constant holomorphic sectional curvature 4 . We consider the Hopf fibration $\bar{\pi}$ :

$$
S^{1} \rightarrow S^{2 n+1} \stackrel{\bar{\pi}}{\longrightarrow} P_{n}(\boldsymbol{C})
$$

where $S^{k}$ denotes the Euclidean sphere of curvature 1 . In $S^{2 n+1}$ we have the family of generalized clifford surfaces whose fibres lie in complex subspaces (see [2]):

$$
M_{2 p+1,2 q+1}=s^{2 p+1}\left(\frac{2 n}{2 p+1}\right) \times s^{2 q+1}\left(\frac{2 n}{2 q+1}\right)
$$

where $p+q=n-1$. Then we have a fibration $\pi$ :

Received 20 February 1984. The author thanks Professor K. Ogiue for his valuable suggestions and encouragement during the preparation of this paper.

Copyright clearance Centre, Inc. Serial-fee code: 0004-9727/84 $\$ A 2.00+0.00$ 


$$
S^{1} \rightarrow M_{2 p+1,2 q+1} \stackrel{\pi}{\longrightarrow} M_{p, q}^{\mathbb{C}}
$$

which is compatible with $\bar{\pi}$.

These manifolds $M_{p, q}^{C}$ thus obtained have various beautiful properties (cf. [3], [4]). In the special case of $p=0, M_{0, n-1}^{\mathbb{L}}$ is called the geodesic minimal hypersphere of $P_{n}(\mathbb{C})$ (see [5]).

Kon ([1]) characterized $M_{0, n-1}$ in terms of sectional curvatures.

The purpose of this paper is to prove a pinching theorem in terms of Ricci curvatures. We have the following

THEOREM. Let $M$ be a connected real minimal hypersurface of $P_{n}(\boldsymbol{C})$. If $n \geqq 3$ and the Ricci curvature $S$ of $M$ satisfies $2 n-2 \leqq S \leqq 2 n$, then $M$ is locally congment to $M_{p, p}^{\mathcal{L}}(2 p=n-1)$.

\section{Preliminaries}

Let $M$ be a real hypersurface of $P_{n}(\boldsymbol{C})$. In a neighbourhood of each point, we choose a unit normal vector field $N$ in $P_{n}(\boldsymbol{C})$. The Riemannian connections $\widetilde{\nabla}$ in $P_{n}(\mathbb{C})$ and $\nabla$ in $M$ are related by the following formulas for arbitrary vector fields $X$ and $Y$ on $M$ :

$$
\begin{gathered}
\widetilde{\nabla}_{X} Y=\nabla_{X} Y+g(A X, Y) N, \\
\widetilde{\nabla}_{X} N=-A X,
\end{gathered}
$$

where $g$ denotes the Riemannian metric on $M$ induced from the Fubinistudy metric $G$ on $P_{n}(\mathbb{C})$ and $A$ is the (local) second fundamental form of $M$ in $P_{n}(\mathbb{E})$. An eigenvector $X$ of the second fundamental form $A$ is called a principal cumature vector. Also an eigenvalue $r$ of $A$ is called a principal curvature. In what follows, we denote by $V_{r}$ the eigenspace of $A$ with eigenvalue $r$. It is known that $M$ has an almost contact metric structure induced from the complex structure $J$ of $P_{n}(E)$ (cf. [4]) i.e. we have a tensor field $\phi$ of type $(1,1)$ on $M$, given by 
$g(\phi X, Y):=G(J X, Y)$ i.e. $\phi(X)=J X-G(J X, N) N$ for all tangent vectors $X, Y$ of $M$, and depending on the local choice of $N$ - one has the unit tangent vector field $\xi$ and the 1 -form $n$ of $M$ defined by

$$
\xi:=-J N \text { respectively } n(X):=g(\xi, X)=G(J X, N) \text {. }
$$

Then we have

$$
\phi^{2}(X)=-X+n(X) \xi, g(\xi, \xi)=1, \phi \xi=0, n(\xi)=1 .
$$

From the above remark and (1.1), we have easily

$$
\left(\nabla_{X} \phi\right) Y=n(Y) A X-g(A X, Y) \xi,
$$

$$
\nabla_{Y} \xi=\phi A Y
$$

Let $\widetilde{R}$ and $R$ be the curvature tensors of $P_{n}(\mathbb{C})$ and $M$, respectively. since the curvature tensor $\widetilde{R}$ has a nice form, we have the following Gauss and Codazzi equations:

$$
\left\{\begin{aligned}
g(R(X, Y) Z, W) & =g(Y, Z) g(X, W)-g(X, Z) g(Y, W)+g(\phi Y, Z) g(\phi X, W) \\
& -g(\phi X, Z) g(\phi Y, W)-2 g(\phi X, Y) g(\phi Z, W) \\
& +g(A Y, Z) g(A X, W)-g(A X, Z) g(A Y, W)
\end{aligned}\right.
$$

and

$$
\left(\nabla_{X} A\right) Y-\left(\nabla_{Y} A\right) X=n(X) \phi Y-n(Y) \phi X-2 g(\phi X, Y) \xi .
$$

Using (1.3) and (1.6), we get

$$
R_{0}(X, Y)=(2 n+1) g(X, Y)-3 n(X) \cdot n(Y)+(\text { trace } A) g(A X, Y)-g(A X, A Y),
$$

where $R_{0}$ denotes the Ricci tensor of $M$.

Moreover, from (1.3) and (1.7) we obtain

$$
g\left(\left(\nabla_{X} A\right) Y, \xi\right)-g\left(\left(\nabla_{Y} A\right) X, \xi\right)=-2 g(\phi X, Y)
$$




\section{Proof of Theorem}

It follows from the assumption that the immersion is minimal and (1.3) that the equation (1.8) implies

$$
R_{0}(\xi, \xi)=2 n-2-g(A \xi, A \xi)
$$

This, together with $R_{0}(\xi, \xi) \geqq 2 n-2$, shows

$$
A \xi=0 \text {. }
$$

Now, differentiating (2.2) covariantly along $X$ and making use of (1.5), for any $y$ we get

$$
g\left(\left(\nabla_{X} A\right) Y, \xi\right)+g(A \phi A X, Y)=0
$$

Exchanging $X$ and $Y$ in $(2.3)$, we see

$$
g\left(\left(\nabla_{Y} A\right) X, \xi\right)+g(A \phi A Y, X)=0
$$

From (1.9), (2.3) and (2.4), we find $g(A \phi A X-\phi X, Y)=0$ so that

$$
A \phi A X=\phi X
$$

Here and in the sequel, let $X(\perp \xi)$ be a principal curvature vector with eigenvalue $r$ i.e. $X \in V_{p^{*}}$ From (2.5) we obtain $r \cdot A(\phi X)=\phi X$, that is,

$$
A(\phi X)=1 / r \cdot \phi X \text {; that is, } \phi X \in V_{1 / r} \text {. }
$$

(In fact, if $r=0$, then $\phi X=0$, which is a contradiction.)

On the other hand the equation (1.8) shows $R_{0}(X, X)=2 n+1-r^{2} \leqq 2 n$ so that $r^{2} \geqq 1$.

Similarly we have $R_{0}(\phi X, \phi X)=2 n+1-1 / r^{2} \leqq 2 n$ so that $r^{2} \leqq 1$. So we find that $r= \pm 1$.

This, together with the assumption that the immersion is minimal, implies that our real hypersurface $M$ has three constant principal curvatures $\{0, \pm 1\}$ at each point.

Here we recall Takagi's work [5]. He determined all real hypersurfaces in $P_{n}(\boldsymbol{C})(n \geq 3)$ with three constant principal curvatures. 
Due to his work, we conclude that our real hypersurface $M$ is locally congruent to $M_{p, p}^{f}(2 p=n-1)$.

of course the manifold $M_{p, p}^{C}$ satisfies the assumption of our Theorem.

\section{References}

[1] M. Kon, "Real minimal hypersurfaces in a complex projective space", Proc. Amer. Math. Soc. 79 (1980), 285-288.

[2] H. B. Lawson, "Rigidity theorems in rank-1 symmetric spaces", J. Diff. Geom. 4 (1970), 349-357.

[3] Y. Maeda, "On real hypersurfaces of a complex projective space", J. Math. Soc. Japan 28 (1976), 529-540.

[4] M. Okumura, "On some real hypersurfaces of a complex projective space", Trans. Amer. Math. Soc. 212 (1975), 355-364.

[5] R. Takagi, "Real hypersurfaces in a complex projective space with constant principal curvatures I and II", J. Math. Soc. Japan 27 (1975) 43-53 and 507-516.

Department of Mathematics,

Tokyo Metropolitan University,

Tokyo 158,

Japan. 